\title{
LOEWNER MATRIX ORDERING IN ESTIMATION OF THE SMALLEST SINGULAR VALUE*
}

\author{
J.M. PEÑA ${ }^{\dagger}$ AND T. SZULC $\ddagger$
}

\begin{abstract}
In this paper, some new lower bounds for the smallest singular value of a square complex matrix $A$ are derived. A key tool to obtain these bounds is using some Hermitian matrices which are, in the sense of Loewner matrix ordering, below the Hermitian part of $A$ or, more generally, below the Hermitian part of the unitary equivalences of $A$. Two types of new bounds are proposed. The first bound can be applied to matrices with positive diagonal entries and strictly diagonally dominant Hermitian parts. It is always at least as large (never worse) as pure Gersgorin-based bound due to C.R. Johnson [C.R. Johnson. A Gersgorin-type lower bound for the smallest singular value. Linear Algebra Appl., 112:1-7, 1989.]. The other bound is complementary with the first one and it can be effectively applied to matrices whose Hermitian parts are very far from diagonal dominance.
\end{abstract}

Key words. Singular values, Loewner ordering, Gudkov condition, Diagonal dominance.

AMS subject classifications. 65F15, 15A18, 15A42, 15A48.

1. Introduction. It is well known that lower bounds for the smallest singular value $\sigma_{n}(A)$ of an $n \times n$ complex matrix $A$ have many potential theoretical and practical applications. Following [4] and [5], recall that $\sigma_{n}(A)$ indicates not only whether or not $A$ is nonsingular, but also how far (in a unitary invariant norm) from the singular matrices $A$ is. Moreover, it is also a key ingredient in the spectral condition number $\sigma_{1}(A) / \sigma_{n}(A)$ (where $\sigma_{1}(A)$ is the largest singular value of $A$ ), which is commonly used in studying numerical calculations involving $A$. So, lower bounds for the smallest singular value in terms of possible simple functions of the entries of a matrix are of interest.

Several results of this type are available in the literature. We first mention the bound derived by Varah in [13] for matrices which are simultaneously strictly diagonally dominant by rows and columns and bounds for more general classes of matrices obtained by Varga in [14]. Bounds involving the determinant of the matrix as well as

*Received by the editors on August 28, 2010. Accepted for publication on April 30, 2011. Handling Editor: Panayiotis Psarrakos.

†Departamento de Matemática Aplicada. Universidad de Zaragoza, 50009 Zaragoza, Spain (jmpena@unizar.es). Supported by the Spanish Research grant MTM2009-07315, and by Gobierno de Aragón and Fondo Social Europeo.

${ }^{\ddagger}$ Faculty of Mathematics and Computer Science, Adam Mickiewicz University, Umultowska 87, 61-614 Poznan, Poland. 
the Euclidean norm of its rows and columns were presented in [3] and [11]. Due to the relationship with our results, we also mention Gersgorin-based bounds given in [6], [7] and [9] and bounds based on some variants of Gersgorin theorem proposed in [8]. In this paper we present some further simple lower bounds for the smallest singular value of a square complex matrix $A$. To get them we make use of the known fact that the smallest singular value of a matrix $A$ is greater than or equal to the smallest eigenvalue of the Hermitian part $H(A)$ of $A$ and focus on Hermitian matrices which are, in the sense of Loewner matrix ordering, below $H(A)$.

In Section 2, we present a condition for a square real matrix to be a $P$-matrix and we recall some basic information on the Loewner matrix ordering. In Sections 3 and 4 , we develop two different applications of this ordering to the problem of bounding the smallest singular value. These applications are complementary and require a computational cost of $\mathcal{O}\left(n^{2}\right)$ elementary operations to bound the smallest singular value of an $n \times n$ matrix.

In Section 3, we derive Gudkov-type bounds for the smallest singular value. We show that these bounds are sharper than those of [8] for the matrices considered therein. A limitation of the proposed bounds follows from the fact that they provide nontrivial results only for matrices which, by means of unitary transformations, become matrices whose Hermitian parts are strictly diagonally dominant. In Section 4 , we obtain the bounds that are complementary with the bounds from Section 3 and which can be applied to more general matrices. These bounds, as shown in an example, can also improve bounds obtained in [6] and [8]. The paper is closed with conclusions presented in Section 5.

2. $H(A)$-type bounds for $\sigma_{n}(A)$. For a given complex matrix $A=\left(a_{i j}\right)_{1 \leq i, j \leq n}$, we define

$$
P_{k}(A)=\sum_{j \neq k}\left|a_{k j}\right|, \quad Q_{k}(A)=\sum_{j \neq k}\left|a_{j k}\right|, \quad k=1, \ldots, n,
$$

and the Hermitian part of $A$, i.e., the matrix

$$
H(A)=\left(\frac{1}{2}\left(a_{i j}+\bar{a}_{j i}\right)\right)_{1 \leq i, j \leq n} .
$$

A matrix $A=\left(a_{i j}\right)_{1 \leq i, j \leq n}$ is said to be strictly diagonally dominant (by rows) if, for each $k=1, \ldots, n,\left|a_{k k}\right|>P_{k}(A)$.

Denote the singular values of $A$ and real eigenvalues of $H(A)$ by $\sigma_{i}(A)$ and $\lambda_{i}(H(A))(i=1, \ldots, n)$, respectively, and assume that:

$$
\sigma_{1}(A) \geq \sigma_{2}(A) \geq \cdots \geq \sigma_{n}(A), \quad \lambda_{1}(H(A)) \geq \lambda_{2}(H(A)) \geq \cdots \geq \lambda_{n}(H(A)) .
$$


It is well-known (Corollary 3.1 .5 of [5]) that

$$
\sigma_{n}(A) \geq \lambda_{n}(H(A))
$$

and that

$$
\lambda_{n}(H(A)) \leq \operatorname{Re}(\lambda) \leq \lambda_{1}(H(A))
$$

hold for any eigenvalue $\lambda$ of $A$ (5 of Section 14-2 of [2]).

Applying Gersgorin theorem to the matrix $H(A)$, where $A$ is a square complex matrix such that $H(A)$ is positive definite, the bound (2.2) becomes

$$
\sigma_{n}(A) \geq \min _{1 \leq k \leq n}\left\{\operatorname{Re}\left(a_{k k}\right)-P_{k}(H(A))\right\} .
$$

In fact, (2.4) is given in Theorem 1 in [7]. This formula provides a nontrivial bound for $\sigma_{n}(A)$ whenever $H(A)$ is strictly diagonally dominant with positive diagonal entries. Of course, as singular values of a Hermitian positive definite matrix coincide with its eigenvalues, and taking into account (2.2), this bound can be obviously extended to derive a lower bound $\sigma_{n}(B) \geq \sigma_{n}(A)$ for any complex matrix $B$ such that $A:=$ $H(U B V)$ is positive definite strictly diagonally dominant for some unitary matrices $U, V$.

Let us notice that Gersgorin theorem was also used in Theorem 3 of [7] to derive the following bound for $\sigma_{n}(A): \sigma_{n}(A) \geq \min _{1 \leq k \leq n}\left\{\left|a_{k k}\right|-\frac{1}{2}\left(P_{k}(A)+Q_{k}(A)\right)\right\}$. It is easy to see that, for matrices with positive diagonal entries, bound (2.4) is always at least as large as the bound from the aforementioned theorem and it is essentially better for $A$ such that $\frac{1}{2}\left(P_{r}(A)+Q_{r}(A)\right)>P_{r}(H(A))$, where $r$ is the index for which the right hand-side in the latter bound (the bound proposed in [7]) attends the minimum. The following example illustrates this fact.

EXAMPLE 2.1. Let

$$
A=\left(\begin{array}{cc}
3 & 2 \\
-2 & 3
\end{array}\right)
$$

Then the bound of Theorem 3 of [7] gives $\sigma_{2}(A) \geq 1$ and, since $H(A)$ is a diagonal matrix with diagonal entries equal to 3 , bound (2.4) gives $\sigma_{2}(A) \geq 3$. In this case $\sigma_{2}(A)=\sqrt{13}$.

A real square matrix such that all its principal minors are positive is called a $P$ matrix. The following result collects the above inequalities relating to singular values and eigenvalues and also provides a class of $P$-matrices.

Proposition 2.2. If $A$ is a square real matrix with positive diagonal entries such that $H(A)$ is positive definite, then $A$ is a P-matrix, and (2.2), (2.3), and

$$
\sigma_{n}(A) \geq \min _{1 \leq k \leq n}\left\{a_{k k}-P_{k}(H(A))\right\}
$$


(see (2.1)) hold.

Proof. Formulae (2.2), (2.3) and (2.5) follow from the hypotheses and the previous comments.

To show that $A$ is a $P$-matrix suppose that $B$ is a principal submatrix of $A$ of order $k, 1 \leq k \leq n$. Clearly, $H(B)$ coincides with the corresponding principal submatrix of $H(A)$. Then, by hypothesis, $H(B)$ is positive definite and so $0<\lambda_{n}(H(B)) \leq \operatorname{Re}(\mu)$ for any eigenvalue $\mu$ of $B$. Hence $\operatorname{det} B>0$ and the result follows.

It should be pointed out that Proposition 2.2 can also be applied to matrices whose Hermitian parts belong to a class of $P$-matrices different from the class of strictly diagonally dominant with positive diagonal entries (for instance, the class of $B$-matrices introduced in [10]).

Further lower bounds for $\sigma_{n}(A)$, sharing a similar type of information on $A$, were derived in [8]. We derive a new bound in Section 3 that improves these bounds in all examples considered in [8].

Observe that, by Corollary 3.1.5 of [5], $\sigma_{1}(A) \geq \lambda_{1}(H(A))$ and so, lower bounds for $\sigma_{1}(A)$ could be derived in a similar way to those for $\sigma_{n}(A)$.

We shall close this section by recalling the definition of Loewner ordering and a basic result of it. Given two Hermitian matrices $A, B$, we write $B \preceq A$ if $A-B$ is positive semidefinite. The partial ordering on the class of Hermitian matrices induced by $\preceq$ is called the Loewner ordering. The following result is well-known (Corollary 4.3 .3 of [4]).

TheOREm 2.3. Let $A, B$ be two Hermitian matrices with eigenvalues $\lambda_{1}(A) \geq$ $\lambda_{2}(A) \geq \cdots \geq \lambda_{n}(A)$ and $\lambda_{1}(B) \geq \lambda_{2}(B) \geq \cdots \geq \lambda_{n}(B)$. If $A \preceq B$, then $\lambda_{k}(A) \leq$ $\lambda_{k}(B), k=1, \ldots, n$.

In the next two sections, we shall present two different applications of Loewner ordering to derive new lower bounds of the smallest singular value of a matrix.

3. A new Gudkov-type bound for the smallest singular value. We start this section by introducing some notations related to Gudkov's condition for nonsingularity. Let $A=\left(a_{i j}\right)_{1 \leq i, j \leq n}$ and let $R_{1}(A):=P_{1}(A)$ (see (2.1)). Then we define for each $i=2, \ldots, n$

$$
R_{i}(A):=\sum_{k=1}^{i-1}\left|a_{i k}\right| \frac{R_{k}(A)}{\left|a_{k k}\right|}+\sum_{k=i+1}^{n}\left|a_{i k}\right|
$$

Before stating Gudkov's nonsingularity condition [1] we mention that matrices that satisfy it are known in the literature as Nekrasov matrices ([12]). 
TheOREM 3.1. Let $A=\left(a_{i j}\right)_{1 \leq i, j \leq n}$ be a complex matrix such that $\left|a_{i i}\right|>R_{i}(A)$ for $i=1, \ldots, n$. Then $A$ is nonsingular.

The following result is an immediate consequence of Theorem 2 of [12].

TheOREM 3.2. Let $A=\left(a_{i j}\right)_{1 \leq i, j \leq n}$ be a complex matrix with positive diagonal entries satisfying $a_{i i}>R_{i}(A)$ for $i=1, \ldots, n$. Then all eigenvalues of $A$ have positive real parts.

For our purposes, we will need the following weaker version of the previous result:

TheOREM 3.3. Let $A=\left(a_{i j}\right)_{1 \leq i, j \leq n}$ be a complex matrix with nonnegative diagonal entries satisfying $a_{i i} \geq R_{i}(A)$ for $i=1, \ldots, n$. Then all eigenvalues of $A$ have nonnegative real parts.

Proof. Let $\varepsilon>0$ and consider the matrix $A_{\varepsilon}:=A+\varepsilon I$. Then one can check that the positive diagonal entries of $A_{\varepsilon}$ satisfy $a_{i i}+\varepsilon>R_{i}(A) \geq R_{i}\left(A_{\varepsilon}\right)$, and so, by Theorem 3.2, all eigenvalues of $A_{\varepsilon}$ have positive real parts. Hence $\operatorname{Re}\left(\lambda_{i}\right)+\varepsilon>0$ for any eigenvalue $\lambda_{i}$ of $A$. Taking into account that these inequalities hold for any $\varepsilon>0$, the result follows.

Now we are ready to present our main result for Hermitian strictly diagonally dominant matrices. To state it the following notion of the last landing index is needed.

Let $\left\{b_{i}\right\}_{i=1, \ldots, n}$ be a nonincreasing ordered sequence of real numbers. The index $j$ such that $b_{j}>b_{j+1}=b_{j+2}=\cdots=b_{n}$ will be called the ll-index (last landing index) for $\left\{b_{i}\right\}_{i=1, \ldots, n}$. If the elements of the sequence coincide, then we set $j:=n$; otherwise, $j$ belongs to $\{1, \ldots, n-1\}$.

TheOREM 3.4. Let $A$ be an $n \times n$ Hermitian matrix strictly diagonally dominant with positive diagonal entries and let $F$ be a permutation matrix such that $\tilde{A}=\left(\tilde{a}_{r s}\right)_{1 \leq r, s \leq n}:=F A F^{T}$ satisfies

$$
\tilde{a}_{11}-P_{1}(\tilde{A}) \geq \tilde{a}_{22}-P_{2}(\tilde{A}) \geq \cdots \geq \tilde{a}_{n n}-P_{n}(\tilde{A})=: w
$$

(see (2.1)) and $\tilde{a}_{i i} \geq \tilde{a}_{k k}$ if $i>k$ and $\tilde{a}_{i i}-P_{i}(\tilde{A})=\tilde{a}_{k k}-P_{k}(\tilde{A})$. Let $j$ be the ll-index of $\left\{\tilde{a}_{i i}-P_{i}(\tilde{A})\right\}_{i=1, \ldots, n}$, let $z:=\tilde{a}_{j j}-P_{j}(\tilde{A})$ and set $v:=w$ if $j=n$ and $v:=\min \{(w+$ $\left.z) / 2, \tilde{a}_{j+1, j+1}\right\}$ otherwise. Let $D$ be the diagonal matrix $D=\operatorname{diag}\left\{d_{1}, \ldots, d_{n}\right\}$ with $d_{i}:=v$ for $i \leq j$ and, for all $i>j, d_{i}:=\tilde{a}_{i i}-R_{i}\left(\tilde{A}-D_{i}\right)$ (see (3.1)), where $D_{i}:=$ $\operatorname{diag}\left\{d_{1}, \ldots, d_{i-1}, 0 \ldots, 0\right\}$. Then the smallest singular value $\sigma_{n}(A)$ of $A$ satisfies

$\sigma_{n}(A) \geq \min \left\{v, \tilde{a}_{j+1, j+1}-R_{j+1}(\tilde{A}-D), \tilde{a}_{j+2, j+2}-R_{j+2}(\tilde{A}-D), \ldots, \tilde{a}_{n n}-R_{n}(\tilde{A}-D)\right\}$.

Proof. We shall prove that $\tilde{A}-D$ is positive semidefinite. As $j$ is the ll-index of 
$\left\{\tilde{a}_{i i}-P_{i}(\tilde{A})\right\}_{i=1, \ldots, n}$ and $v \leq(w+z) / 2$, we have

$$
\tilde{a}_{i i}-v \geq \tilde{a}_{i i}-\frac{w+z}{2} \geq \tilde{a}_{i i}-\left(\tilde{a}_{i i}-P_{i}(\tilde{A})\right)=P_{i}(\tilde{A})=P_{i}(\tilde{A}-D) \geq R_{i}(\tilde{A}-D),
$$

for any index $i$ with $1 \leq i \leq j$, and

$$
\tilde{a}_{i i}-d_{i}=\tilde{a}_{i i}-\left(\tilde{a}_{i i}-R_{i}\left(\tilde{A}-D_{i}\right)\right)=R_{i}\left(\tilde{A}-D_{i}\right)=R_{i}(\tilde{A}-D),
$$

for any index $i$ with $i>j$. So, $\tilde{A}-D$ satisfies the hypotheses of Theorem 3.3 and therefore, as a Hermitian matrix, $\tilde{A}-D$ is positive semidefinite. Then $D \preceq \tilde{A}$ and by Theorem 2.3 , the smallest eigenvalue of $\tilde{A}$ is greater than or equal to the smallest eigenvalue of $D$, which is its smallest diagonal entry. Since $\lambda_{n}(\tilde{A})=\lambda_{n}(A)=\sigma_{n}(A)$, we obtain (3.2).

If all rows of $A$ have the same diagonal dominance (i.e., if $a_{11}-P_{1}(A)=\cdots=$ $\left.a_{n n}-P_{n}(A)\right)$, then the previous result leads again to the bound (2.5).

Let us particularize Theorem 3.4 in the important case in which there exists a unique row with the minimal strict diagonal dominance.

Corollary 3.5. Let $A$ be a matrix satisfying the hypotheses of Theorem 3.4 with $j=n-1$. Then, with the notation of Theorem 3.4, the smallest singular value $\sigma_{n}(A)$ of $A$ satisfies

$$
\sigma_{n}(A) \geq \min \left\{v, \tilde{a}_{n n}-R_{n}(\tilde{A}-D)\right\}
$$

Our next result applies Theorem 3.4 to non-Hermitian matrices.

Corollary 3.6. Let $B$ be an $n \times n$ complex matrix such that $A:=H(U B V)$ is positive definite strictly diagonally dominant for some unitary matrices $U, V$. Then the smallest singular value $\sigma_{n}(B)$ of $B$ satisfies

$\sigma_{n}(B) \geq \min \left\{v, \tilde{a}_{j+1, j+1}-R_{j+1}(\tilde{A}-D), \tilde{a}_{j+2, j+2}-R_{j+2}(\tilde{A}-D), \ldots, \tilde{a}_{n n}-R_{n}(\tilde{A}-D)\right\}$,

where $v, \tilde{A}=\left(\tilde{a}_{r s}\right)_{1 \leq r, s \leq n}, j$, and $D$ are defined as in Theorem 3.4.

Proof. Since $U, V$ are unitary matrices, $\sigma_{n}(U B V)=\sigma_{n}(B)$ and (3.3) follows by comment on page 3 and by applying Theorem 3.4 to $A=H(U B V)$.

Let us particularize the previous result in the important case in which the Hermitian part possesses a unique row with the minimal strict diagonal dominance.

Corollary 3.7. Let $B$ be a matrix satisfying the hypotheses of Corollary 3.6 with $j=n-1$. Then the smallest singular value $\sigma_{n}(B)$ of $B$ satisfies

$$
\sigma_{n}(B) \geq \min \left\{v, \tilde{a}_{n n}-R_{n}(\tilde{A}-D)\right\},
$$


where $v, \tilde{A}=\left(\tilde{a}_{i j}\right)_{1 \leq i, j \leq n}$ and $D$ are defined as in Theorem 3.4 .

REMARK 3.8. Theorem 3.4 and Corollary 3.6 still hold if we replace the choice $v:=\min \left\{(w+z) / 2, \tilde{a}_{j+1, j+1}\right\}$ by $v:=\min \left\{(w+z) / 2, \tilde{a}_{n n}\right\}$, as it can be checked from their proofs. Although $v$ is smaller with this choice, the remaining terms of the right-hand sides of the bounds (3.2) and (3.3) can be greater.

REMARK 3.9. Observe that forming the matrix $A:=H(U B V)$ requires $\left(n^{2}-n\right) / 2$ sums and divisions, calculating the diagonal dominance of each row of $A$ to transform it into $\tilde{A}$ requires $n^{2}-2 n$ sums, $n$ subtractions and $\mathcal{O}(n \log n)$ comparisons and obtaining $v$ requires 1 sum, 1 division and $n-j+1$ comparisons. Moreover, forming $D, \tilde{A}-D$, obtaining $R_{n}(\tilde{A}-D)$, calculating the differences $\tilde{a}_{j+1, j+1}-R_{j+1}(\tilde{A}-$ $D), \tilde{a}_{j+2, j+2}-R_{j+2}(\tilde{A}-D), \ldots, \tilde{a}_{n n}-R_{n}(\tilde{A}-D)$ and checking Gudkov's condition requires $\mathcal{O}\left(n^{2}\right)$ elementary operations. Finally, additional $n-j+1$ comparisons are needed. So, the computational cost for obtaining the bound of Corollary 3.6 for the smallest singular value of an $n \times n$ matrix if $U B V$ is known has the order of $\mathcal{O}\left(n^{2}\right)$ elementary operations. Example 4.7 illustrates how $U, V$ can be chosen in practice.

REMARK 3.10. Similarly as in the proof of Theorem 3.4 one can show that a strictly diagonally dominant Hermitian A with positive diagonal entries is, in the Loewner ordering, over $D=\operatorname{diag}(d, \ldots, d)$, where $d=\min _{k}\left\{a_{k k}-P_{k}(A)\right\}$ and in consequence (see Corollary 3.6), for $B$ satisfying the hypotheses of Corollary 3.6, obtain the bound (2.4).

Remark 3.11. Following Remark 3.10 and the definition of $R_{i}(A)$, it is easy to see that for a matrix $B$ with positive diagonal entries satisfying the hypotheses of Corollary 3.6, the bound (3.3) is always at least as large as the bound (2.4) (and therefore at least as large as the bound given in Theorem 3 of [7]) and for $B$ such that all sums of the first $i-1$ terms in $P_{i}(H(U B V)), i=2, \ldots, n$, are nonzero the bound (3.3) is essentially better than the bound (2.4) (and therefore essentially better than the bound given in Theorem 3 of [7]). Moreover, if $B$ satisfies the hypotheses of Corollary 3.6 and $r$ is the index for which the right hand-side in the bound (2) from [8] attends the minimum, our experiments show that, when $P_{r}(B)$ is "close" to $Q_{r}(B)$, the bound (3.3) is better than the bound (2) of [8].

We shall close this section giving examples which illustrate efficiency of our results. We consider the matrices used in Section 5 of [8] to compare the best bounds for the smallest singular value. Our new bounds will always improve bounds obtained in [8] for these examples. 
ExAmple 3.12. Let us start with the matrices $A_{1}$ and $A:=H\left(A_{1}\right)$ with

$$
A_{1}=\left(\begin{array}{ccc}
224 & 21 & 55 \\
61 & 137 & 66 \\
-83 & -26 & 175
\end{array}\right), A=\left(\begin{array}{ccc}
224 & 41 & -14 \\
41 & 137 & 20 \\
-14 & 20 & 175
\end{array}\right), \tilde{A}=\left(\begin{array}{ccc}
224 & -14 & 41 \\
-14 & 175 & 20 \\
41 & 20 & 137
\end{array}\right)
$$

Then, with the notations of Theorem 3.4, we have $z=141, w=76$ and also $v=$ $\min \left\{137, \frac{217}{2}\right\}=\frac{217}{2}=d_{1}=d_{2}$. So we have $R_{1}(\tilde{A}-D)=P_{1}(\tilde{A}-D)=55$, $R_{2}(\tilde{A}-D)=\frac{1540}{231}+20=\frac{6160}{231}$ and $R_{3}(\tilde{A}-D)=\frac{4510}{231}+\frac{246400}{30723} \approx 27.54$. Then $d_{3}=\tilde{a}_{33}-$ $R_{3}(\tilde{A}-D) \approx 109.46$ and the bound of Corollary 3.6 is $\sigma_{3}\left(A_{1}\right) \geq \min \{108.5,109.46\}=$ 108.5. Let us observe that $\sigma_{3}\left(A_{1}\right)=117.83$ and the best bound found in [8] was 55.72. Let us now consider the matrices $A_{2}$ and $A:=H\left(A_{2}\right)$ with

$$
A_{2}=\left(\begin{array}{ccc}
259 & -9 & 44 \\
-18 & 94 & -2 \\
-43 & 8 & 243
\end{array}\right), A=\left(\begin{array}{ccc}
259 & -\frac{27}{2} & \frac{1}{2} \\
-\frac{27}{2} & 94 & 3 \\
\frac{1}{2} & 3 & 243
\end{array}\right), \tilde{A}=\left(\begin{array}{ccc}
259 & \frac{1}{2} & -\frac{27}{2} \\
\frac{1}{2} & 243 & 3 \\
-\frac{27}{2} & 3 & 94
\end{array}\right)
$$

Then, with the notations of Theorem 3.4, we have $z=\frac{479}{2}, w=\frac{155}{2}$ and also $v=$ $\min \left\{94, \frac{634}{4}\right\}=94=d_{1}=d_{2}$. So we have $R_{1}(\tilde{A}-D)=P_{1}(\tilde{A}-D)=14, R_{2}(\tilde{A}-D)=$ $\frac{7}{165}+3=\frac{502}{165}$ and $R_{3}(\tilde{A}-D)=\frac{189}{165}+\frac{1506}{24585} \approx 1.20$. Then $d_{3}=\tilde{a}_{33}-R_{3}(\tilde{A}-D) \approx 92.80$ and the bound of Corollary 3.6 is $\sigma_{3}\left(A_{2}\right) \geq \min \{94,92.80\}=92.80$. Let us observe that $\sigma_{3}\left(A_{2}\right)=92.88$ and the best bound found in [8] was 87.84 .

The last matrix studied in [8] was $A_{3}$ :

$$
A_{3}=\left(\begin{array}{ccc}
10 & 1 & 1 \\
1 & 20 & 1 \\
1 & 1 & 30
\end{array}\right), \quad A:=H\left(A_{3}\right)=A_{3}, \quad \tilde{A}=\left(\begin{array}{ccc}
30 & 1 & 1 \\
1 & 20 & 1 \\
1 & 1 & 10
\end{array}\right) .
$$

Then, with the notations of Theorem 3.4, we have that $z=18, w=8$ and $v=$ $\min \{10,13\}=10=d_{1}=d_{2}$. So we have $R_{1}(\tilde{A}-D)=P_{1}(\tilde{A}-D)=2, R_{2}(\tilde{A}-D)=$ $\frac{1}{10}+1=\frac{11}{10}$ and $R_{3}(\tilde{A}-D)=\frac{1}{10}+\frac{11}{100}=0.21$. Then $d_{3}=\tilde{a}_{33}-R_{3}(\tilde{A}-D)=9.79$ and the bound of Corollary 3.6 is $\sigma_{3}\left(A_{3}\right) \geq \min \{10,9.79\}=9.79$. Let us observe that $\sigma_{3}\left(A_{3}\right)=9.86$ and the best bound found in [8] was 9.64 .

4. Complementary results to bound the smallest singular value. In this section, we present another application of the Loewner ordering that complements the results of the previous section. Let us recall (Definition 2.5.11 in [5]) that an $n \times n$ complex matrix $A=\left(a_{i j}\right)_{1 \leq i, j \leq n}$ is said to be strictly diagonally dominant of its row entries if $\left|a_{i i}\right|>\left|a_{i j}\right|$ for each $i=1, \ldots, n$ and all $j \neq i$.

THEOREM 4.1. Let $M=\left(m_{i j}\right)_{1 \leq i, j \leq n}$ be a Hermitian matrix strictly diagonally dominant of its row entries and with positive diagonal. If there exists $0<c(M)<$ $\min _{1 \leq i \leq n}\left\{m_{i i}\right\}$ such that the matrix $C:=M-c(M)(1, \ldots, 1)^{T}(1, \ldots, 1)$ is strictly diagonally dominant with positive diagonal entries, then $\sigma_{n}(M) \geq \sigma_{n}(C)$. 
Proof. Observe that the Hermitian matrix $M-C$ is positive semidefinite because its eigenvalues are 0 (with multiplicity $n-1$ ) and $n c(M)>0$. So, $C \preceq M$ and by Theorem 2.3, the smallest eigenvalue of $M$ (which coincides with $\sigma_{n}(M)$ ) is greater than or equal to the smallest eigenvalue of $C$ (which coincides with $\sigma_{n}(C)$ ).

Corollary 4.2. Let $A=\left(a_{i j}\right)_{1 \leq i, j \leq n}$ be an $n \times n$ complex matrix such that $M:=H(U A V)$ is a matrix characterized in Theorem 4.1 for some unitary matrices $U, V$. Then the smallest singular value $\sigma_{n}(A)$ of $A$ satisfies $\sigma_{n}(A) \geq \sigma_{n}(C)$, where $C$ is defined in Theorem 4.1, and for lower bounds for $\sigma_{n}(C)$ one can use the bounds obtained in Sections 2 and 3.

Proof. As $U, V$ are unitary matrices, $\sigma_{n}(A)=\sigma_{n}(U A V)$ and the assertion follows from Theorem 4.1.

The efficiency of the results of this section depends on the spectral properties of the matrix $C$, and the following remark provides some suggestions on the choice of the number $c(M)$.

REMARK 4.3. As $c(M)$ is positive, in order to reach our purposes (i.e., either the matrix $C$ becomes strictly diagonally dominant or its strict row diagonal dominance is increased) it is necessary that the number $q$ of nonpositive off-diagonal entries of $M$ satisfies $2 q<n(n-1)$ since otherwise we increase the sum of the absolute values of the off-diagonal entries. In this case, a good choice to diminish the positive offdiagonal entries and to control the increasing of the absolute values of the remaining off-diagonal entries is the choice $c(M):=m$, where $m$ is the arithmetic mean of the off-diagonal entries. Let us mention two exceptions for this choice:

- $m$ does not satisfy $0<m<\min _{1 \leq i \leq n}\left\{m_{i i}\right\}$,

- $2(q+r(s)) \geq n(n-1)$, where $s$ is the least positive off-diagonal entry of $M$ and $r(s)$ denotes the number of off-diagonal entries of $M$ equal to $s$.

In the first case we recommend the choice $c(M):=s$ and in the second case the choice $c(M):=\min \{m, s\}$. Finally, let us observe that the choice of $c(M)$ proposed here increases the computational cost considered in Remark 3.9 in at most $\left(n^{2}-n-\right.$ $2) / 2$ sums, one division and at most $\left(n^{2}-n\right) / 2+1$ comparisons. The computation of the matrix

$$
C=M-c(M)(1, \ldots, 1)^{T}(1, \ldots, 1)
$$

requires $\left(n^{2}+n\right) / 2$ elementary operations. Thus we still keep a computational cost of $\mathcal{O}\left(n^{2}\right)$ elementary operations for an $n \times n$ matrix, as in Remark 3.9.

We shall close Section 4 by examples illustrating efficiency of Theorem 4.1 and Corollary 4.2 . 
EXAMPLE 4.4. Let

$$
M=\left(\begin{array}{cccccc}
10 & 5 & 4 & 3 & 2 & 1 \\
5 & 10 & 3 & 2 & 1 & 4 \\
4 & 3 & 10 & 1 & 5 & 2 \\
3 & 2 & 1 & 10 & 4 & 5 \\
2 & 1 & 5 & 4 & 10 & 3 \\
1 & 4 & 2 & 5 & 3 & 10
\end{array}\right)
$$

with $\sigma_{6}(M)=2.6411$. According to Remark 4.3, the choice of $c(M)$ is $c(M)=m$, where $m=3$ is the arithmetic mean of the off-diagonal entries. Then, by Theorem 4.1 and denoting by $G:=c(M)(1, \ldots, 1)^{T}(1, \ldots, 1)$, we can write

$$
M=C+G=\left(\begin{array}{cccccc}
7 & 2 & 1 & 0 & -1 & -2 \\
2 & 7 & 0 & -1 & -2 & 1 \\
1 & 0 & 7 & -2 & 2 & -1 \\
0 & -1 & -2 & 7 & 1 & 2 \\
-1 & -2 & 2 & 1 & 7 & 0 \\
-2 & 1 & -1 & 2 & 0 & 7
\end{array}\right)+\left(\begin{array}{cccccc}
3 & \ldots & \ldots & \ldots & \cdots & 3 \\
\vdots & & & & & \vdots \\
\vdots & & & & & \vdots \\
\vdots & & & & & \vdots \\
3 & \ldots & \ldots & \ldots & \ldots & 3
\end{array}\right),
$$

and $\sigma_{6}(M) \geq \sigma_{6}(C) \geq 1$ by $(2.5)$. Let us observe that $\sigma_{6}(C)=1.028$. With the choice of $c(M)=s=1$ as the least positive off-diagonal entry of $M$, the corresponding matrix $C=M-G$ is not strictly diagonally dominant. Observe that, by Corollary 4.2 , we also have $\sigma_{6}(A) \geq \sigma_{6}(C) \geq 1$ for the matrix

$$
A=\left(\begin{array}{cccccc}
10 & 12 & 5 & 9 & 2 & 3 \\
-2 & 10 & 4 & 5 & 2 & 10 \\
3 & 2 & 10 & 2 & 7 & -2 \\
-3 & -1 & 0 & 10 & 6 & 5 \\
2 & 0 & 3 & 2 & 10 & 6 \\
-1 & -2 & 6 & 5 & 0 & 10
\end{array}\right)
$$

since $H(A)=M\left(\sigma_{6}(A)=5.4345\right)$.

In contrast to Theorem 3.4, Theorem 4.1 can be applied to matrices that are very far from diagonal dominance, as the following example shows.

EXAMPLE 4.5. For any $n$ odd, let us consider the following $n \times n$ matrix $M=$ $\left(m_{i j}\right)_{1 \leq i, j \leq n}$ :

$$
M=\left(\begin{array}{cccccc}
2 n & n-1 & n+1 & \cdots & n-1 & n+1 \\
n-1 & 2 n & n-1 & \cdots & n+1 & n-1 \\
\vdots & & \ddots & & & \vdots \\
\vdots & & & \ddots & & \vdots \\
n-1 & n+1 & n-1 & \cdots & 2 n & n-1 \\
n+1 & n-1 & n+1 & \cdots & n-1 & 2 n
\end{array}\right)
$$


with diagonal entries $m_{i i}=2 n$ and off-diagonal entries $m_{i j}=n-1$ if $i+j$ is odd and $m_{i j}=n+1$ if $i+j$ is even. In this case, since one half of the off-diagonal entries coincide with the least positive off-diagonal entry $s=n-1$ of $M$, the choice of $c(M)$ corresponding to Remark 4.3 is $c(M)=s=n-1=\min \{m, s\}$, and so, by Theorem 4.1 , we can write $M=C+c(M)(1, \ldots, 1)^{T}(1, \ldots, 1)$ :

$$
\begin{aligned}
& \left(\begin{array}{cccccc}
n+1 & 0 & 2 & \cdots & 0 & 2 \\
0 & n+1 & 0 & \cdots & 2 & 0 \\
\vdots & & \ddots & & & \vdots \\
\vdots & & & \ddots & & \vdots \\
0 & 2 & 0 & \cdots & n+1 & 0 \\
2 & 0 & 2 & \cdots & 0 & n+1
\end{array}\right)+\left(\begin{array}{cccccc}
n-1 & \cdots & \cdots & \cdots & \cdots & n-1 \\
\vdots & & & & & \vdots \\
\vdots & & & & & \vdots \\
\vdots & & & & & \vdots \\
\vdots & & & & & \vdots \\
n-1 & \cdots & \cdots & \cdots & \cdots & n-1
\end{array}\right), \\
& C=\left(\begin{array}{cccccc}
n+1 & 0 & 2 & \cdots & 0 & 2 \\
0 & n+1 & 0 & \cdots & 2 & 0 \\
\vdots & & \ddots & & & \vdots \\
\vdots & & & \ddots & & \vdots \\
0 & 2 & 0 & \cdots & n+1 & 0 \\
2 & 0 & 2 & \cdots & 0 & n+1
\end{array}\right)
\end{aligned}
$$

Then $\sigma_{n}(M) \geq \sigma_{n}(C) \geq 2$ by (2.5). With the choice $c(M)=m$, where $m=n$ is the arithmetic mean of the off-diagonal entries, the corresponding matrix

$$
C=M-c(M)(1, \ldots, 1)^{T}(1, \ldots, 1)=\left(\begin{array}{cccccc}
n & -1 & 1 & \cdots & -1 & 1 \\
-1 & n & -1 & \cdots & 1 & -1 \\
\vdots & & \ddots & & & \vdots \\
\vdots & & & \ddots & & \vdots \\
-1 & 1 & -1 & \cdots & n & -1 \\
1 & -1 & 1 & \cdots & -1 & n
\end{array}\right)
$$

and $\sigma_{n}(M) \geq \sigma_{n}(C) \geq 1$ by $(2.5)$.

In our next example we consider a matrix that has both positive and negative entries.

EXAMPLE 4.6. Let

$$
M=\left(\begin{array}{ccc}
7 & 5 & 3 \\
5 & 10 & -2 \\
3 & -2 & 10
\end{array}\right)
$$

with $\sigma_{3}(M)=1.7145$. In this case, $2(q+r(s))=2(2+2) \geq 3(3-1)=6$ and therefore, following Remark 4.3 (see (ii)), we choose $c(M)=\min \{m, s\}=\min \{2,3\}=2$. So, 
by Theorem 4.1 , we can write

$$
M=\left(\begin{array}{ccc}
5 & 3 & 1 \\
3 & 8 & -4 \\
1 & -4 & 8
\end{array}\right)+\left(\begin{array}{lll}
2 & 2 & 2 \\
2 & 2 & 2 \\
2 & 2 & 2
\end{array}\right)
$$

and $\sigma_{3}(M) \geq \sigma_{3}(C)$. By $(2.5), \sigma_{3}(C) \geq 1$. In fact, $\sigma_{3}(C)=1.5472$. With the choice $c(M)=s=3$, the corresponding matrix $C=M-c(M)(1, \ldots, 1)^{T}(1, \ldots, 1)$ is not strictly diagonally dominant.

An easy application of Corollary 4.2 appears when $U$ and/or $V$ are permutation matrices. Since most of the off-diagonal entries should be positive as commented in Remark 4.3, and in fact the bounds are better if we increase the number of positive off-diagonal entries, we can apply the previous results with signature matrices in addition to the permutation matrices. The following example comes from Example 2 of Section 17-12 of [2] and illustrates this idea.

EXAMPLE 4.7. Let

$$
B=\left(\begin{array}{cccc}
11 & -3 & -5 & 1 \\
1 & -5 & -3 & 11 \\
-5 & 1 & 11 & -3 \\
-3 & 11 & 1 & -5
\end{array}\right)
$$

Let $F$ be the permutation matrix such that $B F$ exchanges columns 2 and 4 of $B$, so that $B F$ is strictly diagonally dominant with positive diagonal entries (and Hermitian). Using the signature matrix $S=\operatorname{diag}\{1,1,-1,-1\}$ we obtain

$$
M:=S(B F) S=\left(\begin{array}{cccc}
11 & 1 & 5 & 3 \\
1 & 11 & 3 & 5 \\
5 & 3 & 11 & 1 \\
3 & 5 & 1 & 11
\end{array}\right)
$$

According to Remark 4.3, the choice of $c(M)$ is $c(M)=3$, i.e. $c(M)$ is equal to the arithmetic mean of the off-diagonal entries. Then, by Theorem 4.1 , we can write

$$
M=C+c(M)(1, \ldots, 1)^{T}(1, \ldots, 1)=\left(\begin{array}{cccc}
8 & -2 & 2 & 0 \\
-2 & 8 & 0 & 2 \\
2 & 0 & 8 & -2 \\
0 & 2 & -2 & 8
\end{array}\right)+\left(\begin{array}{cccc}
3 & 3 & 3 & 3 \\
3 & 3 & 3 & 3 \\
3 & 3 & 3 & 3 \\
3 & 3 & 3 & 3
\end{array}\right)
$$

and $\sigma_{4}(B)=\sigma_{4}(M) \geq \sigma_{4}(C) \geq 4$ by $(2.5)$, which is in fact optimal because $\sigma_{4}(B)=4$ (see Example 2 of Section 17-12 of [2]).

The fifth example of this section shows that our bounds can improve those presented in [6] (and again bounds from [8]). 
EXAMPLE 4.8. Let

$$
A=\left(\begin{array}{ccc}
0.75 & 0.5 & 0.4 \\
0.5 & 1 & 0.6 \\
0 & 0.5 & 1
\end{array}\right)
$$

be the matrix considered in Example 4 of [6]. Then, by Theorem 2 therein, we get $\sigma_{3}(A) \geq 0.056$ (the true value of the minimal singular value is $\sigma_{3}(A)=0.2977$ ). Observe that $M:=H(A)$ satisfies

$$
M=\left(\begin{array}{ccc}
0.75 & 0.5 & 0.2 \\
0.5 & 1 & 0.55 \\
0.2 & 0.55 & 1
\end{array}\right)=C+\left(\begin{array}{ccc}
0.2 & 0.2 & 0.2 \\
0.2 & 0.2 & 0.2 \\
0.2 & 0.2 & 0.2
\end{array}\right), \quad C=\left(\begin{array}{ccc}
0.55 & 0.3 & 0 \\
0.3 & 0.8 & 0.35 \\
0 & 0.35 & 0.8
\end{array}\right) \text {. }
$$

Hence, by (2.3), Theorem 4.1 and (2.6), $\sigma_{3}(A) \geq \sigma_{3}(M) \geq \sigma_{3}(C) \geq 0.15$ (note that, by Theorem 3 from [8], we get the bound 0.0086).

We have compared our bounds with others sharing a similar type of information on a matrix. However, our bounds can also improve the bounds having "a different nature" than ours, as those of [11]. For example, for a $3 \times 3$ diagonal matrix $D=$ $\operatorname{diag}\left(d_{1}, d_{2}, d_{3}\right)$ with $d_{1} \geq d_{2} \geq d_{3}>0$, the bound $(22)$ of $[11]$ is $\sigma_{3}(D)>(2 / 3) d_{3}$ and the bound (2.5) gives $\sigma_{3}(D) \geq d_{3}$, which is in fact an equality.

5. Conclusions. This paper deals with lower bounds for the smallest singular value $\sigma_{n}(A)$ of a complex square matrix $A$. We use the fact that the smallest singular value of a matrix is not less than the smallest eigenvalue of its Hermitian part, which is combined with well known result about Loewner ordering. Theorem 3.4 from Section 3 gives an estimation for Hermitian matrices strictly diagonally dominant with positive diagonal entries, and consequently for (up to permutation) matrices, Hermitian parts of which have this property. If any main diagonal entry is not positive, it is easy to remedy it since $\sigma_{n}(A)$ is a unitarily invariant function of $A$. In particular, if all diagonal entries of $A$ are nonzero then, after multiplication of $A$ by diagonal matrix $D$ with the entries equal to the quotients of the conjugate of the entry and its modulus, the diagonal entries of $D A$ will be positive. In Section 4 the result is generalized to the class of matrices obtained from the previous one by appropriate rank-one perturbations.

The presented bounds are simple, in the sense that they can be computed easily and require a computational cost of $\mathcal{O}\left(n^{2}\right)$ elementary operations to bound the smallest singular value of an $n \times n$ matrix. Numerical examples illustrate the sharpness of the proposed bounds, comparing to known ones. Although results of Section 3 can be applied only to matrices such that their Hermitian part can be transformed into a strictly diagonally dominant matrix by means of unitary transformation, the results of Section 4 can be applied to matrices whose Hermitian parts are very far from diagonal dominance. 
Acknowledgment. The authors are grateful to the referees for constructive comments and suggestions that helped to improve the presentation.

\section{REFERENCES}

[1] V.V. Gudkov. On a certain test for non-singularity of matrices (Russian). In Latvian Math. Yearbook, Izdat. "Zinatne", Riga, 385-390, 1965 (MR 33, 1966, \#1323).

[2] L. Hogben (Ed.). Handbook of Linear Algebra. Discrete Mathematics and its Applications (Boca Raton). Chapman \& Hall/CRC, Boca Raton, FL, 2007.

[3] Y.P. Hong and C.T. Pan. A lower bound for the smallest singular value. Linear Algebra Appl., 172:27-32, 1992

[4] R.A. Horn and C.R. Johnson. Matrix Analysis. Cambridge University Press, Cambridge, 1985.

[5] R.A. Horn and C.R. Johnson. Topics in Matrix Analysis, Cambridge University Press, Cambridge, 1991.

[6] T.-Z. Huang. Estimation of $\left\|A^{-1}\right\|_{\infty}$ and the smallest singular value. Comput. Math. Appl., $55: 1075-1080,2008$.

[7] C.R. Johnson. A Gersgorin-type lower bound for the smallest singular value. Linear Algebra Appl., 112:1-7, 1989.

[8] C.R. Johnson and T. Szulc. Further lower bounds for the smallest singular value. Linear Algebra Appl., 272:169-179, 1998 .

[9] C.R. Johnson, T. Szulc, and D. Wojtera-Tyrakowska. Optimal Gersgorin-style estimation of extremal singular values. Linear Algebra Appl., 402:46-60, 2005.

[10] J.M. Peña. A class of $P$-matrices with applications to the localization of the eigenvalues of a real matrix. SIAM J. Matrix Anal. Appl., 22:1027-1037, 2001 .

[11] O. Rojo. Further bounds for the smallest singular value and the spectral condition number. Comput. Math. Appl., 38:215-228, 1999.

[12] T. Szulc. Some remarks on a theorem of Gudkov. Linear Algebra Appl., 225:221-235, 1995.

[13] J.M. Varah. A lower bound for the smallest singular value of a matrix. Linear Algebra Appl., 11:3-5, 1975 .

[14] R.S. Varga. On diagonal dominance arguments for bounding $\left\|A^{-1}\right\|_{\infty}$. Linear Algebra Appl., 14:211-217, 1976. 